\title{
Stability of Gabor Frames Under Small Time Hamiltonian Evolutions
}

\author{
MAURICE A. DE GOSSON, KARLHEINZ GRÖCHENIG \\ and JOSÉ LUIS ROMERO \\ Faculty of Mathematics, University of Vienna, Oskar-Morgenstern-Platz 1, 1090 Vienna, \\ Austria.e-mail: maurice.de.gosson@univie.ac.at
}

Received: 2 November 2015 / Revised: 13 April 2016 / Accepted: 14 April 2016

Published online: 6 May 2016 - (C) The Author(s) 2016. This article is published with open access at Springerlink.com

\begin{abstract}
We consider Hamiltonian deformations of Gabor systems, where the window evolves according to the action of a Schrödinger propagator and the phase-space nodes evolve according to the corresponding Hamiltonian flow. We prove the stability of the frame property for small times and Hamiltonians consisting of a quadratic polynomial plus a potential in the Sjöstrand class with bounded second-order derivatives. This answers a question raised in de Gosson (Appl Comput Harmonic Anal 38(2):196-221, 2015)
\end{abstract}

Mathematics Subject Classification. 35Q70, 35Q41, 42C40, 81S10, 81S30.

Keywords. time-frequency analysis, Gabor frame, Hamiltonian flow, Schrödinger equation, Weyl quantization, phase space, Wigner distribution.

\section{Introduction}

Let $\mathcal{H}(x, p)$ be a Hamiltonian on $\mathbb{R}^{2 d}$ and $H:=\mathcal{H}^{w}$ its Weyl quantization. The solution to the Schrödinger equation

$$
\begin{aligned}
& i \partial_{t} u(t, \cdot)=H u(t, \cdot), \quad t \in \mathbb{R}, \\
& u(0, \cdot)=f
\end{aligned}
$$

is given by the propagation formula $u(t, \cdot)=e^{-i t H} f$. The model case is the one of a real quadratic (homogeneous) Hamiltonian: $\mathcal{H}(x, p)=\langle M(x, p),(x, p)\rangle$, with $M \in \mathbb{R}^{2 d \times 2 d}$ being symmetric. In this case, the evolution operator $e^{-i t H}$ and the (symmetric) time-frequency shift operators

M. d. G. was supported by the grant P 23902 from the Austrian Science Fund (FWF). K. G. was supported in part by the project P26273-N25 of the Austrian Science Fund (FWF). J. L. R. gratefully acknowledges support from a Marie Curie fellowship, within the 7th European Community Framework program, under grant PIIF-GA-2012-327063. 


$$
\rho(z) f:=e^{-\pi i x \xi} e^{2 \pi i \xi} f(\cdot-x), \quad z=(x, \xi) \in \mathbb{R}^{2 d},
$$

satisfy the symplectic covariance relation

$$
e^{-i t H} \rho(z)=\rho\left(e^{2 t J M} z\right) e^{-i t H},
$$

where $J=\left(\begin{array}{cc}0 & I \\ -I & 0\end{array}\right)$ is the standard symplectic form (see for example [11, Chapter 15]). Thanks to (2), the action of the evolution operator on a state $f$ can be understood by considering an expansion into coherent states:

$$
f=\sum_{\lambda \in \Lambda} c_{\lambda} \rho(\lambda) g
$$

where $g$ is a smooth, fast decaying function, $\Lambda \subseteq \mathbb{R}^{d}$ is a set of phase-space nodes and $c_{\lambda} \in \mathbb{C}$. Such an expansion is a discrete version of the continuous coherent state representation [2], and the canonical choice for $g$ is a Gaussian function. The evolution generated by the quadratic Hamiltonian $\mathcal{H}$ is then given by

$$
e^{-i t H} f=\sum_{\lambda \in \Lambda} c_{\lambda} \rho\left(e^{2 t J M} \lambda\right) e^{-i t H} g,
$$

and therefore the description of the evolution of an arbitrary state $f$ is reduced to the one of $g$. If $\mathcal{H}(x, p)=x^{2}+p^{2}$ is the harmonic oscillator and $g$ is chosen to be an adequate Gaussian function, then $e^{-i t H} g=g$, and (4) amounts to a rearrangement of the time-frequency content of $f$. The case of higher-order Hermite functions is also important, since these correspond to higher-energy Landau levels (see [1]).

The collection of coherent states

$$
\mathcal{G}(g, \Lambda):=\{\rho(\lambda) g: \lambda \in \Lambda\}
$$

is called a Gabor system, and it is a Gabor frame if every $f \in L^{2}\left(\mathbb{R}^{d}\right)$ admits an expansion as in (3) with $\|c\|_{2} \asymp\|f\|_{2}$. In this case, several properties of $f$ can be read from the coefficients $c$. The theory of Gabor frames-also called Weyl-Heisenberg frames - plays an increasingly important role in physics; see for instance $[9,13,11]$ and the references therein.

Recently, one of us started the investigation of the relation between the theory of Gabor frames and Hamiltonian and quantum mechanics [12] and introduced the notion of a Hamiltonian deformation of a Gabor system. For a (time-independent) Hamiltonian $\mathcal{H}(x, p)$, we let $\Phi_{t}(x, p)$ be the flow given by the Hamilton equations

$$
\left\{\begin{array}{l}
\dot{x}=\mathcal{H}_{p}(x, p), \\
\dot{p}=-\mathcal{H}_{x}(x, p),
\end{array}\right.
$$

and let $H:=\mathcal{H}^{w}$ be the Weyl quantization of $\mathcal{H}$. Given a Gabor system $\mathcal{G}(g, \Lambda)$, we consider the time-evolved systems

$$
\mathcal{G}_{t}(g, \Lambda):=\mathcal{G}\left(e^{-i t H} g, \Phi_{t} \Lambda\right)
$$


and investigate the stability of the frame property under the evolution $\mathcal{G}(g, \Lambda) \mapsto$ $\mathcal{G}_{t}(g, \Lambda)$.

When $\mathcal{H}$ is a quadratic form $\mathcal{H}(x, p)=\langle M(x, p),(x, p)\rangle$, its flow is given by the linear map $\Phi_{t}(x, p)=e^{2 t J M}(x, p)$ and (2) expresses the fact that the evolution operator $e^{-i t H}$ is the metaplectic operator associated with the linear map $e^{2 t J M}$. As a consequence, $\mathcal{G}_{t}(g, \Lambda)$ is the image of $\mathcal{G}(g, \Lambda)$ under the unitary map $e^{-i t H}$ and hence it enjoys the same spanning properties (in particular, the frame property is preserved). This observation is called the symplectic covariance of Gabor frames [12].

For more general Hamiltonians $\mathcal{H}$, no strict covariance property holds, and the analysis of the deformation $t \mapsto \mathcal{G}_{t}(g, \Lambda)$ is difficult. In [12], one of us analyzed a linearized version of this problem and established some stability estimates (see also [6] for a higher-order approximation to the deformation problem). Based on these results, [12] conjectured that the evolution $\mathcal{G}(g, \Lambda) \mapsto \mathcal{G}_{t}(g, \Lambda)$ preserves the frame property for more general Hamiltonians. In particular, one would expect perturbations of quadratic Hamiltonians to exhibit a certain approximate symplectic covariance, in the form of stability of the frame property of $\mathcal{G}_{t}(g, \Lambda)$ for a certain range of time.

In this article, we solve the deformation problem for small times. More precisely, we consider a perturbation of a quadratic Hamiltonian by an element of the Sjöstrand class $M^{\infty, 1}\left(\mathbb{R}^{2 d}\right)$ with bounded second-order derivatives. We also consider a Gabor frame with window in the Feichtinger algebra $M^{1}\left(\mathbb{R}^{d}\right)$ of functions with integrable Wigner distribution. (See Sections 2 and 2.1 for precise definitions). The following is our main result.

THEOREM 1.1. Let a be a real-valued, quadratic, homogeneous polynomial on $\mathbb{R}^{2 d}$ and let $\sigma \in M^{\infty, 1}\left(\mathbb{R}^{2 d}\right) \cap C^{2}\left(\mathbb{R}^{2 d}\right)$ have bounded second-order derivatives. Consider the Hamiltonian $\mathcal{H}(t, x, p):=a(x, p)+\sigma(x, p)$. Let $H:=\mathcal{H}^{w}(x, D)$ be the Weyl quantization of $\mathcal{H}$ and let $\left(\Phi_{t}\right)_{t \in \mathbb{R}}$ be the flow of $\mathcal{H}$.

Let $g \in M^{1}\left(\mathbb{R}^{d}\right)$ and $\Lambda \subseteq \mathbb{R}^{2 d}$, such that $\mathcal{G}(g, \Lambda)$ is a Gabor frame. Then, there exists $t_{0}>0$ such that for all $t \in\left[-t_{0}, t_{0}\right], \mathcal{G}\left(e^{-i t H} g, \Phi_{t}(\Lambda)\right)$ is a Gabor frame.

To see what is at stake, we consider once more the symplectic covariance property (2). It links the classical Hamiltonian flow $e^{2 t J M}$ on phase space to the quantum mechanical evolution. If $\mathcal{H}$ is not quadratic, then the flow $\Phi_{t}$ is no longer linear, and, in general, there is no explicit and exact formula for the quantum mechanical evolution. We therefore have to understand the classical evolution of the set $\Lambda$ under $\Phi_{t}$ separately from the quantum mechanical evolution of the state (window) $g$ under $e^{-i t H}$.

The stability of the frame property of $\mathcal{G}\left(g, \Phi_{t}(\Lambda)\right)$ is part of the deformation theory of Gabor frames. While there is a significant literature on the stability of Gabor frames under linear distortions of the time-frequency nodes $\Lambda$ (covering 
perturbation of lattice parameters $[4,18]$ on the one hand, and general point sets [3]), only recently a fully non-linear deformation theory of Gabor systems was developed in [23]. It turns out that the concept of Lipschitz deformation is precisely the right tool to treat non-linear Hamiltonian flows, and we will use the main result of [23] in a decisive manner.

The second ingredient in Theorem 1.1 is the assumption $g \in M^{1}\left(\mathbb{R}^{d}\right)$. This is an essential assumption for Gabor frames to be useful in phase space analysis. In particular, most stability results for Gabor frames under perturbations of the window require that $g \in M^{1}\left(\mathbb{R}^{d}\right)$. Outside $M^{1}$, one encounters quickly pathologies [10]. With regard to our problem, it is therefore important to understand whether $M^{1}\left(\mathbb{R}^{d}\right)$ is invariant under the evolution of the Schrödinger equation. This is indeed the case for certain classes of Hamiltonians $[5,8]$ and will be the second important tool used to prove Theorem 1.1.

The rest of the article is organized as follows. In Section 2, we provide some definitions and background results. Section 3 collects the essential tools and derives some auxiliary estimates. Finally, the proof of Theorem 1.1 is presented in Section 4.

\section{Background}

\subsection{TIME-FREQuency ANALYSIS}

Given a function $g \in L^{2}\left(\mathbb{R}^{d}\right)$, with $\|g\|_{2}=1$, the short-time Fourier transform of a function $f \in L^{2}\left(\mathbb{R}^{d}\right)$ with respect to the window $g$ is defined as

$$
V_{g} f(x, \xi):=\left\langle f, e^{\pi i x \xi} \rho(x, \xi) g\right\rangle, \quad(x, \xi) \in \mathbb{R}^{2 d},
$$

where $\rho(x, \xi)$ is the (symmetric) time-frequency shift defined in (1). The function $g$ is often called window and the normalization $\|g\|_{2}=1$ implies that

$$
\left\|V_{g} f\right\|_{L^{2}\left(\mathbb{R}^{2 d}\right)}=\|f\|_{L^{2}\left(\mathbb{R}^{d}\right)}, \quad f \in L^{2}\left(\mathbb{R}^{d}\right) .
$$

The standard choice for $g$ is the Gaussian $\phi(x):=2^{d / 4} e^{-\pi|x|^{2}}$. Analogously, the Feichtinger algebra, originally introduced in [14], is defined to be

$$
M^{1}\left(\mathbb{R}^{d}\right):=\left\{f \in L^{2}\left(\mathbb{R}^{d}\right):\|f\|_{M^{1}}:=\left\|V_{\phi} f\right\|_{L^{1}\left(\mathbb{R}^{2 d}\right)}<+\infty\right\}
$$

and is used as a standard reservoir for windows $g$. Equivalently, $f \in M^{1}\left(\mathbb{R}^{d}\right)$, if the Wigner distribution $W f(x, \xi)=\int f(x+t / 2) \overline{f(x-t / 2)} e^{-2 \pi i \xi \cdot t} \mathrm{~d} t$ of $f$ is integrable on $\mathbb{R}^{2 d}$. When $g \in M^{1}\left(\mathbb{R}^{d}\right)$, the map $f \mapsto V_{g} f$ can be extended beyond $L^{2}\left(\mathbb{R}^{d}\right)$.

We define the modulation spaces as follows: fix a non-zero $g \in \mathcal{S}\left(\mathbb{R}^{d}\right)$ and let $1 \leq$ $p, q \leq \infty$. Then, $M^{p, q}\left(\mathbb{R}^{d}\right)$ is the class of all distributions $f \in \mathcal{S}^{\prime}\left(\mathbb{R}^{d}\right)$ such that

$$
\|f\|_{M^{p, q}\left(\mathbb{R}^{d}\right)}:=\left(\int_{\mathbb{R}^{d}}\left(\int_{\mathbb{R}^{d}}\left|V_{g} f(x, \xi)\right|^{p} \mathrm{~d} x\right)^{q / p} \mathrm{~d} \xi\right)^{1 / q}<\infty,
$$


with the usual modification when $p$ or $q$ is $\infty$. Different choices of non-zero windows $g \in \mathcal{S}\left(\mathbb{R}^{d}\right)$ yield the same space with equivalent norms; see [17] and [20, Chapter 11]. In addition, for $g \in M^{1}\left(\mathbb{R}^{d}\right)$, the short-time Fourier transform is well defined on all $M^{p, q}\left(\mathbb{R}^{d}\right)$. Originally introduced by Feichtinger in [15], modulation spaces combine smoothness and integrability conditions. In this article, we will be mainly concerned with Feichtinger's algebra $M^{1}\left(\mathbb{R}^{d}\right)$, as a window class for Gabor systems, and $M^{\infty, 1}\left(\mathbb{R}^{2 d}\right)$ - also known as Sjöstrand's class - as a symbol class for pseudodifferential operators.

\subsection{SAmpling the Short-Time Fourier TRANSForm}

A set $\Lambda \subseteq \mathbb{R}^{d}$ is called relatively separated if

$$
\operatorname{rel}(\Lambda):=\sup \left\{\#\left(\Lambda \cap\left(\{x\}+[0,1]^{d}\right)\right): x \in \mathbb{R}^{d}\right\}<\infty .
$$

The assumption that $g \in M^{1}\left(\mathbb{R}^{d}\right)$ implies certain sampling estimates for the shorttime Fourier transform. We quote the following standard result (see for example [20, Chapter 13].)

PROPOSITION 2.1. Let $g \in M^{1}\left(\mathbb{R}^{d}\right)$ and let $\Lambda \subseteq \mathbb{R}^{2 d}$. Then,

$$
\left(\sum_{\lambda \in \Lambda}\left|V_{g} f(\lambda)\right|^{2}\right)^{1 / 2} \leq C \operatorname{rel}(\Lambda)\|g\|_{M^{1}}\|f\|_{2}, \quad f \in L^{2}\left(\mathbb{R}^{d}\right),
$$

where the constant $C$ depends only on the dimension $d$.

\subsection{GABOR FRAMES}

Given a window $g \in M^{1}\left(\mathbb{R}^{d}\right)$ and a relatively separated set $\Lambda \subseteq \mathbb{R}^{2 d}$, the collection of functions

$$
\mathcal{G}(g, \Lambda):=\{\rho(\lambda) g: \lambda \in \Lambda\}
$$

is called the Gabor system generated by $g$ and $\Lambda$. It is a Gabor frame, if there exist constants $A, B>0$ such that

$$
A\|f\|_{2}^{2} \leq \sum_{\lambda \in \Lambda}|\langle f, \rho(\lambda) g\rangle|^{2} \leq B\|f\|_{2}^{2}, \quad f \in L^{2}\left(\mathbb{R}^{d}\right) .
$$

The constants $A, B$ are called frame bounds for $\mathcal{G}(g, \Lambda)$. We remark that the definition of Gabor system given here is slightly non-standard. In signal processing, it is more common to define the time-frequency shifts by

$$
\pi(z) f(t):=e^{2 \pi i \xi t} f(t-x), \quad z=(x, \xi) \in \mathbb{R}^{d} \times \mathbb{R}^{d}, t \in \mathbb{R}^{d} .
$$

Since $\pi(x, \xi)=e^{\pi i x \xi} \rho(x, \xi)$, the choice $\rho$ has no impact on the frame inequality in (10). Note that the sum in (10) is the same as $\left\|\left.V_{g} f\right|_{\Lambda}\right\|_{2}^{2}$. The use of $\rho$ instead of 
$\pi$ in this article is motivated by the symplectic covariance property in (2), which would require additional phase factors if $\pi$ was used instead of $\rho$.

The following basic fact can be found for example in [7, Theorem 1.1].

LEMMA 2.2. If $\mathcal{G}(g, \Lambda)$ is a frame, then $\Lambda$ is relatively separated.

\section{The Essential Tools}

\subsection{SCHRÖDinger Operators ON MOdULATION SPACES}

The Weyl transform of a distribution $\sigma \in \mathcal{S}^{\prime}\left(\mathbb{R}^{d} \times \mathbb{R}^{d}\right)$ is an operator $\sigma^{w}$ that is formally defined on functions $f: \mathbb{R}^{d} \rightarrow \mathbb{C}$ as

$$
\sigma^{w}(f)(x):=\int_{\mathbb{R}^{d} \times \mathbb{R}^{d}} \sigma\left(\frac{x+y}{2}, \xi\right) e^{2 \pi i(x-y) \xi} f(y) \mathrm{d} y \mathrm{~d} \xi, \quad x \in \mathbb{R}^{d} .
$$

The fundamental results in the theory of pseudodifferential operators provide conditions on $\sigma$ for the operator $\sigma^{w}$ to be well defined and bounded on various function spaces. In particular, Sjöstrand proved that if $\sigma \in M^{\infty, 1}\left(\mathbb{R}^{2 d}\right)$, then $\sigma^{w}$ is bounded on $L^{2}\left(\mathbb{R}^{d}\right)[25,26]$. See also [21,22] for extensions of these results to weighted symbol classes and modulations spaces.

The following result is one of our main tools. It shows that perturbing a quadratic Hamiltonian with a potential in the Sjöstrand's class $M^{\infty, 1}\left(\mathbb{R}^{2 d}\right)$ gives rise to propagators that are strongly continuous on $M^{1}\left(\mathbb{R}^{d}\right)$.

THEOREM 3.1 ([8, Theorems 1.5 and 4.1]). Let a be a real-valued, quadratic, homogeneous polynomial on $\mathbb{R}^{2 d}$ and let $\sigma \in M^{\infty, 1}\left(\mathbb{R}^{2 d}\right)$. Let $H:=a^{w}(x, D)+$ $\sigma^{w}(x, D)$. Then, $e^{i t H}$ is a strongly continuous one-parameter group of operators on $M^{1}\left(\mathbb{R}^{d}\right)$. In other words,

(a) for all $t \in \mathbb{R}, e^{i t H}: M^{1}\left(\mathbb{R}^{d}\right) \rightarrow M^{1}\left(\mathbb{R}^{d}\right)$,

(b) for each $g \in M^{1}\left(\mathbb{R}^{d}\right)$,

$$
e^{i t H} g \longrightarrow g \text { in } M^{1}\left(\mathbb{R}^{d}\right) \text {, as } t \longrightarrow 0 .
$$

\subsection{Deformation of Gabor Frames}

Our second essential tool is a description of the stability of the frame property of a Gabor frame $\mathcal{G}(g, \Lambda)$ under small deformations of $\Lambda$. Our general assumption is that $g \in M^{1}\left(\mathbb{R}^{d}\right)$. (Without this assumption, the frame property might be very unstable under perturbation of $\Lambda$, even for lattices [10,24]).

The classical results in signal processing describe the stability of the frame property under the so-called jitter perturbations: if $\mathcal{G}(g, \Lambda) \sup _{\lambda \in \Lambda} \inf _{\lambda^{\prime} \in \Lambda^{\prime}}\left|\lambda-\lambda^{\prime}\right|<$ $\epsilon$ and $\sup _{\lambda^{\prime} \in \Lambda^{\prime}} \inf _{\lambda \in \Lambda}\left|\lambda-\lambda^{\prime}\right|<\epsilon$, for sufficiently small $\varepsilon$, then $\mathcal{G}\left(g, \Lambda^{\prime}\right)$ is also a frame. A much deeper property is the stability of the frame condition under linear maps $\Lambda \mapsto A \Lambda$, where $A$ is a matrix that is sufficiently close to the identity (but 
possibly not symplectic!). Such results have been derived first for lattices $[4,18]$ and then for general sets [3]. To deal with Hamiltonian flows, we will resort to a recent fully non-linear stability theory [23].

Let $\Lambda \subseteq \mathbb{R}^{d}$ be a set. We consider a sequence $\left\{\Lambda_{n}: n \geq 1\right\}$ of subsets of $\mathbb{R}^{d}$ produced in the following way. For each $n \geq 1$, let $\tau_{n}: \Lambda \rightarrow \mathbb{R}^{d}$ be a map and let $\Lambda_{n}:=$ $\tau_{n}(\Lambda)=\left\{\tau_{n}(\lambda): \lambda \in \Lambda\right\}$. We assume that $\tau_{n}(\lambda) \longrightarrow \lambda$, as $n \longrightarrow \infty$, for all $\lambda \in \Lambda$. The sequence of sets $\left\{\Lambda_{n}: n \geq 1\right\}$ together with the maps $\left\{\tau_{n}: n \geq 1\right\}$ is called a deformation of $\Lambda$. We think of each sequence of points $\left\{\tau_{n}(\lambda): n \geq 1\right\}$ as a (discrete) path moving toward the end point $\lambda$.

We will say that $\left\{\Lambda_{n}: n \geq 1\right\}$ is a deformation of $\Lambda$, with the understanding that a sequence of underlying maps $\left\{\tau_{n}: n \geq 1\right\}$ is also given.

We now describe a special class of deformations.

DEFINITION 3.2. A deformation $\left\{\Lambda_{n}: n \geq 1\right\}$ of $\Lambda$ is called Lipschitz, denoted by $\Lambda_{n} \stackrel{\text { Lip }}{\longrightarrow} \Lambda$, if the following two conditions hold:

(L1) Given $R>0$,

$$
\sup _{\substack{\lambda, \lambda^{\prime} \in \Lambda \\\left|\lambda-\lambda^{\prime}\right| \leq R}}\left|\left(\tau_{n}(\lambda)-\tau_{n}\left(\lambda^{\prime}\right)\right)-\left(\lambda-\lambda^{\prime}\right)\right| \rightarrow 0, \text { as } n \longrightarrow \infty \text {. }
$$

(L2) Given $R>0$, there exists $R^{\prime}>0$ and $n_{0} \in \mathbb{N}$ such that if $\left|\tau_{n}(\lambda)-\tau_{n}\left(\lambda^{\prime}\right)\right| \leq R$ for some $n \geq n_{0}$ and some $\lambda, \lambda^{\prime} \in \Lambda$, then $\left|\lambda-\lambda^{\prime}\right| \leq R^{\prime}$.

The following results shows that the frame property of a Gabor system is stable under Lipschitz deformations.

THEOREM 3.3 ([23, Thm. 7.1 and Rem. 7.3]). Let $g \in M^{1}\left(\mathbb{R}^{d}\right)$ and $\Lambda \subseteq \mathbb{R}^{2 d}$. Assume that $\mathcal{G}(g, \Lambda)$ is a (Gabor) frame and that $\Lambda_{n} \stackrel{\text { Lip }}{\longrightarrow} \Lambda$. Then, there exist $A, B>0$ and $n_{0} \in \mathbb{N}$ such that $\mathcal{G}\left(g, \Lambda_{n}\right)$ is a frame with uniform bounds $A, B$ for all $n \geq n_{0}$.

We will also need the following technical lemma concerning Lipschitz convergence and relative separation.

LEMMA 3.4 ([23, Lemma 6.7]). Let $\Lambda_{n} \stackrel{\text { Lip }}{\longrightarrow} \Lambda$ and assume that $\Lambda$ is relatively separated. Then, $\lim \sup _{n} \operatorname{rel}\left(\Lambda_{n}\right)<\infty$.

The following corollary enables us to combine the stability of Gabor frames under deformations of $\Lambda$ with small perturbations of the window $g$ on $M^{1}$-norm. 
COROLlARY 3.5. Assume that $g_{n} \longrightarrow g$ in $M^{1}\left(\mathbb{R}^{d}\right)$ and that $\Lambda_{n} \stackrel{\text { Lip }}{\longrightarrow} \Lambda$. Then, $\mathcal{G}\left(g_{n}, \Lambda_{n}\right)$ is a frame for all sufficiently large $n$. (Moreover, the corresponding frame bounds can be taken to be uniform in $n$ ).

Proof. By Theorem 3.3, there exist $A, B>0$ and $n_{0} \in \mathbb{N}$ such that for all $n \geq n_{0}$,

$$
A\|f\|_{2} \leq\left\|V_{g} f \mid \Lambda_{n}\right\|_{2} \leq B\|f\|_{2}, \quad f \in L^{2}\left(\mathbb{R}^{d}\right) .
$$

(Here, $A, B$ are the square roots of the frame bounds.) By Lemma 2.2, $\Lambda$ and all $\Lambda_{n}$ with $n \gg 0$ are relatively separated. Using Proposition 2.1, we deduce that for all $f \in L^{2}\left(\mathbb{R}^{d}\right)$

$$
\left|\left\|V_{g} f\left|\Lambda_{n}\left\|_{2}-\right\| V_{g_{n}} f\right| \Lambda_{n}\right\|_{2}\right| \leq\left\|V_{g-g_{n}} f \mid \Lambda_{n}\right\|_{2} \leq C\left\|g-g_{n}\right\|_{M^{1}} \operatorname{rel}\left(\Lambda_{n}\right)\|f\|_{2} .
$$

Letting

$$
\begin{aligned}
& A_{n}:=A-C\left\|g-g_{n}\right\|_{M^{1}} \operatorname{rel}\left(\Lambda_{n}\right), \\
& B_{n}:=B+C\left\|g-g_{n}\right\|_{M^{1}} \operatorname{rel}\left(\Lambda_{n}\right),
\end{aligned}
$$

we deduce from (12) and the triangle inequality that

$$
A_{n}\|f\|_{2} \leq\left\|V_{g_{n}} f \mid \Lambda_{n}\right\|_{2} \leq B_{n}\|f\|_{2}, \quad f \in L^{2}\left(\mathbb{R}^{d}\right) .
$$

By Lemma 3.4 and the fact that $g_{n} \longrightarrow g$ in $M^{1}$, it follows that $A_{n} \longrightarrow A$ and $B_{n} \longrightarrow B$. Combining this with (13) we conclude that for all sufficiently large $n$,

$$
A / 2\|f\|_{2} \leq\left\|V_{g_{n}} f \mid \Lambda_{n}\right\|_{2} \leq B / 2\|f\|_{2}, \quad f \in L^{2}\left(\mathbb{R}^{d}\right) .
$$

Hence, for $n \gg 1, \mathcal{G}\left(g_{n}, \Lambda_{n}\right)$ is a frame with bounds $A^{2} / 4, B^{2} / 4$.

\subsection{Flows AND LIPSCHITZ CONVERGENCE}

A function $F: \mathbb{R} \times \mathbb{R}^{d} \rightarrow \mathbb{R}^{d}$ is Lipschitz in the second variable if there exists $L>0$ such that

$$
|F(t, x)-F(t, y)| \leq L|x-y|, \quad \text { for all } \quad(t, x) \in \mathbb{R} \times \mathbb{R}^{d} .
$$

Under this assumption, we let $\left(\Phi_{t}\right)_{t \in \mathbb{R}}$ denote the flow of $F$ (associated with time $0)$. This means that for each $x \in \mathbb{R}^{d}, \mathbb{R} \ni t \mapsto \Phi_{t}(x) \in \mathbb{R}^{d}$ is a $C^{1}$ function and that

(a) $\Phi_{0}(x)=x$,

(b) $\frac{d}{d t} \Phi_{t}(x)=F\left(t, \Phi_{t}(x)\right)$.

The theory of ODEs implies that the flow exists and it is uniquely determined by properties (a) and (b) above. Moreover, the flow satisfies the following distortion estimate: given $T>0$, there exist constants $c_{t}, C_{T}>0$ such that

$$
c_{T}|x-y| \leq\left|\Phi_{t}(x)-\Phi_{t}(y)\right| \leq C_{T}|x-y|, \quad x, y \in \mathbb{R}, \quad t \in[-T, T] .
$$

The previous estimate is normally proved using the following useful lemma. 
LEMMA 3.6 (Gronwall). Let $I \subseteq \mathbb{R}$ be an interval and $a \in I$. Let $g: I \rightarrow[0,+\infty)$ be a continuous function that satisfies

$$
g(t) \leq A+B\left|\int_{a}^{t} g(s) \mathrm{d} s\right|, \quad t \in I,
$$

for some constants $A, B \in \mathbb{R}$. Then,

$$
g(t) \leq A e^{B|t-a|}, \quad t \in I .
$$

(The reason for the absolute value outside the integral is that $t-a$ can be negative.)

We now show that the flows of ODEs provide examples of Lipschitz deformations.

THEOREM 3.7. Let $F: \mathbb{R} \times \mathbb{R}^{d} \rightarrow \mathbb{R}^{d}$ be Lipschitz in the second variable and let $\left(\Phi_{t}\right)_{t \in \mathbb{R}}$ be the corresponding flow. Let $\Lambda \subseteq \mathbb{R}^{d}$ be a relatively separated set. Then, $\Phi_{t}(\Lambda) \stackrel{\text { Lip }}{\longrightarrow} \Lambda$, as $t \longrightarrow 0$.

(More precisely, for each sequence $t_{n} \longrightarrow 0, \Phi_{t_{n}}(\Lambda) \stackrel{\text { Lip }}{\longrightarrow} \Lambda$.)

Proof. Let $L>0$ be the Lipschitz constant of $F$ (in the second variable). We first check condition $(L 1)$ from Definition 3.2. From the definition of the flow, it follows that

$$
\Phi_{t}(x)=x+\int_{0}^{t} F\left(s, \Phi_{s}(x)\right) \mathrm{d} s, \quad t \in \mathbb{R} .
$$

Therefore,

$$
\Phi_{t}(\lambda)-\Phi_{t}\left(\lambda^{\prime}\right)-\left(\lambda-\lambda^{\prime}\right)=\int_{0}^{t}\left(F\left(s, \Phi_{s}(\lambda)\right)-F\left(s, \Phi_{s}\left(\lambda^{\prime}\right)\right)\right) \mathrm{d} s .
$$

As a consequence,

$$
\begin{aligned}
\left|\Phi_{t}(\lambda)-\Phi_{t}\left(\lambda^{\prime}\right)-\left(\lambda-\lambda^{\prime}\right)\right| & \leq\left|\int_{0}^{t}\right| F\left(s, \Phi_{s}(\lambda)\right)-F\left(s, \Phi_{s}\left(\lambda^{\prime}\right)\right)|\mathrm{d} s| \\
& \leq\left|\int_{0}^{t} L\right| \Phi_{s}(\lambda)-\Phi_{s}\left(\lambda^{\prime}\right)|\mathrm{d} s| \\
& \leq L\left|\int_{0}^{t}\right| \Phi_{t}(\lambda)-\Phi_{t}\left(\lambda^{\prime}\right)-\left(\lambda-\lambda^{\prime}\right)|\mathrm{d} s|+L|t|\left|\lambda-\lambda^{\prime}\right| .
\end{aligned}
$$

Applying Gronwall's Lemma 3.6 to $g(t):=\left|\Phi_{t}(\lambda)-\Phi_{t}\left(\lambda^{\prime}\right)-\left(\lambda-\lambda^{\prime}\right)\right|$, we deduce that

$$
\left|\Phi_{t}(\lambda)-\Phi_{t}\left(\lambda^{\prime}\right)-\left(\lambda-\lambda^{\prime}\right)\right| \leq L|t|\left|\lambda-\lambda^{\prime}\right| e^{L|t|} .
$$

Condition (L1) follows from here. 
To check condition (L2), we consider only $t \in[-1,1]$ and (14) to obtain a constant $C$ such that

$$
C^{-1}|x-y| \leq\left|\Phi_{t}(x)-\Phi_{t}(y)\right| \leq C|x-y|, \quad t \in(-1,1) .
$$

Hence, if for some instant $t_{0}$ we know that $\left|\Phi_{t_{0}}(\lambda)-\Phi_{t_{0}}\left(\lambda^{\prime}\right)\right| \leq R$, then we can deduce that $\left|\lambda-\lambda^{\prime}\right| \leq R^{\prime}:=C R$.

This completes the proof.

\section{Hamiltonian Deformations: Dénouement}

We finally combine all tools from the previous section and prove the main result.

Proof of Theorem 1.1. Let us define $F: \mathbb{R} \times \mathbb{R}^{2 d} \rightarrow \mathbb{R}^{2 d}$ by

$$
F(t, x, p):=\left(\partial_{p} \mathcal{H}(x, p),-\partial_{x} \mathcal{H}(x, p)\right) .
$$

Then, $F$ is a $C^{1}$ function with bounded derivatives and, consequently, $F$ is Lipschitz in the second set of variables $(x, p)$. Let $t_{n} \longrightarrow 0$ and define $\Lambda_{n}:=\Phi_{t_{n}}(\Lambda)$. Theorem 3.7 implies that $\Lambda_{n} \stackrel{\text { Lip }}{\longrightarrow} \Lambda$, while Theorem 3.1 implies that $e^{-i t_{n} H} g \longrightarrow g$ in $M^{1}$. Hence, Corollary 3.5 yields the desired conclusion.

Remark 4.1. The proof shows that, under the conditions of Theorem 1.1, the Gabor systems $\mathcal{G}\left(e^{-i t h} g, \Phi_{t}(\Lambda)\right)$ admit uniform frame bounds for $t \in\left[-t_{0}, t_{0}\right]$.

Remark 4.2. We do not know whether the conclusion of Theorem 1.1 remains valid for arbitrary times. Moreover, we do not know of any example of a Hamiltonian deformation that does not preserve the frame property.

Open Access This article is distributed under the terms of the Creative Commons Attribution 4.0 International License (http://creativecommons.org/licenses/by/4.0/), which permits unrestricted use, distribution, and reproduction in any medium, provided you give appropriate credit to the original author(s) and the source, provide a link to the Creative Commons license, and indicate if changes were made.

\section{References}

1. Abreu, L.D., Feichtinger, H.G.: Function spaces of polyanalytic functions. In: Harmonic and complex analysis and its applications, Trends Math., pp. 1-38. Birkhäuser/Springer, Cham (2014)

2. Ali, S., Antoine, J.-P., Gazeau, J.-P.: Coherent states, wavelets and their generalizations. Graduate texts in contemporary physics. Springer, New York (2000)

3. Ascensi, G., Feichtinger, H.G., Kaiblinger, N.: Dilation of the Weyl symbol and BalianLow theorem. Trans. Am. Math. Soc. 366(7), 3865-3880 (2014)

4. Bellissard, J.: Lipshitz continuity of gap boundaries for Hofstadter-like spectra. Commun. Math. Phys. 160(3), 599-613 (1994) 
5. Bényi, Á., Gröchenig, K., Okoudjou, K.A., Rogers, L.G.: Unimodular Fourier multipliers for modulation spaces. J. Funct. Anal. 246(2), 366-384 (2007)

6. Berra, M., Bulai, M., Cordero, E., Nicola, F.: Gabor Frames of Gaussian beams for the Schrödinger equation. Appl. Comput. Harmonic Anal. (2016, To appear). doi:10. 1016/j.acha.2015.11.001

7. Christensen, O., Deng, B., Heil, C.: Density of Gabor frames. Appl. Comput. Harmonic Anal. 7(3), 292-304 (1999)

8. Cordero, E., Gröchenig, K., Nicola, F., Rodino, L.: Generalized metaplectic operators and the Schrödinger equation with a potential in the Sjöstrand class. J. Math. Phys. 55, 081506 (2014). doi:10.1063/1.4892459

9. Cordero, E., Nicola, F., Rodino, L.: Propagation of the Gabor wave front set for Schrödinger equations with non-smooth potentials. Rev. Math. Phys. 27(1), 1550001, 33 (2015)

10. Dai, X.R., Sun, Q.: The abc problem for Gabor systems. arXiv:1304.7750

11. de Gosson, M.: Symplectic methods in harmonic analysis and in mathematical physics. Pseudo-differential operators theory and applications, vol 7. Birkhäuser/Springer Basel AG, Basel (2011)

12. de Gosson, M.: Hamiltonian deformations of Gabor frames: first steps. Appl. Comput. Harmonic Anal. 38(2), 196-221 (2015)

13. Doran, R.S., Moore, C.C., Zimmer, R.J.: Group representations, ergodic theory, and mathematical physics: a tribute to George W. Mackey: AMS Special Session Honoring the Memory of George W. Mackey, January 7-8 (2007) New Orleans, Louisiana. Contemporary Mathematics, American Mathematical Soc (2008)

14. Feichtinger, H.G.: On a new Segal algebra. Monatsh. Math. 92, 269-289 (1981)

15. Feichtinger, H.G.: Modulation spaces on locally compact Abelian groups. Technical report (1983)

16. Feichtinger, H.G.: Banach convolution algebras of Wiener type. In: Sz.-Nagy, B., Szabados, J., (eds.) Proc. Conf. on Functions, Series, Operators, Budapest 1980, Colloq. Math. Soc. Janos Bolyai, vol. 35, pp. 509-524. North-Holland, Amsterdam (1983)

17. Feichtinger, H,G.: Modulation spaces: looking back and ahead. Sampl. Theory Signal Image Process. 5(2), 109-140 (2006)

18. Feichtinger, H.G., Kaiblinger, N.: Varying the time-frequency lattice of Gabor frames. Trans. Am. Math. Soc. 356(5), 2001-2023 (2004)

19. Folland, G.B.: Harmonic analysis in phase space. Princeton Univ. Press, Princeton (1989)

20. Gröchenig, K.: Foundations of time-frequency analysis. Appl. Numer. Harmon. Anal. Birkhäuser, Boston (2001)

21. Gröchenig, K.: Composition and spectral invariance of pseudodifferential operators on modulation spaces. J. Anal. Math. 98, 65-82 (2006)

22. Gröchenig, K.: Time-frequency analysis of Sjörstrand's class. Rev. Mat. Iberoam. 22(2), 703-724 (2006)

23. Gröchenig, K., Ortega-Cerdà, J., Romero, J.L.: Deformation of Gabor systems. Adv. Math. 277(4), 388-425 (2015)

24. Janssen, A.J.E.M.: On generating tight Gabor frames at critical density. J. Fourier Anal. Appl. 9(2), 175-214 (2003)

25. Sjöstrand, J.: An algebra of pseudodifferential operators. Math. Res. Lett. 1(2), 185192 (1994)

26. Sjöstrand, J.: Wiener type algebras of pseudodifferential operators. Séminaire sur les équations aux Dérivées Partielles, 1994-1995, École Polytech, Palaiseau, Exp. No. IV, p. 21 (1995) 